\title{
Intervención interdisciplinaria del paciente con dolor en Unidades de Cuidado Intensivo (UCI): Revisión de tema
}

\author{
Interdisciplinary intervention of the patient with \\ pain in Intensive Care Units (ICU): Review
}

\begin{abstract}
Ximena Palacios-Espinosa ${ }^{1}$, Jessica Pulido-Rozo ${ }^{1}$, Diana Ramos-Zamudio ${ }^{1}$
Forma de citar: Palacios-Espinosa X, Pulido-Rozo, J, Ramos-Zamudio D. Intervención interdisciplinaria del paciente con dolor en Unidades de Cuidado Intensivo (UCI): revisión de tema. Rev Univ Ind Santander Salud. 49(2): 380-390.

DOI: http://dx.doi.org/10.18273/revsal.v49n2-2017010 @ (])

\section{RESUMEN}

El dolor es una condición frecuente en los pacientes que se encuentran en Unidades de Cuidado Intensivo (UCI), precipitado y mantenido por estímulos biológicos, psicológicos y sociales. Por esta razón, la atención del paciente con dolor en estos escenarios, trasciende el alcance médico y requiere de la interdisciplinariedad. Los objetivos de esta revisión fueron presentar las estrategias de evaluación e intervención del dolor en pacientes que se encuentran en UCI y proponer una estrategia de abordaje interdisciplinario del dolor en estas unidades. La literatura muestra que los métodos de evaluación del dolor son diversos y se utilizan instrumentos específicos cualitativos y cuantitativos según el estado de consciencia del paciente. Estos métodos pueden ser utilizados por diversos profesionales. La intervención del dolor en la UCI está a cargo básicamente de los profesionales de la Enfermería y la Medicina, a pesar de las evidentes necesidades psicológicas de los pacientes. Con base en la evidencia de que la intervención del paciente dolor en UCI es multidisciplinaria, se propone una intervención interdisciplinaria que incluya a la familia del paciente.
\end{abstract}

Palabras clave: Dolor, Unidades de Cuidados Intensivos, dimensión del dolor, terapia conductista, manejo del dolor.

\begin{abstract}
Pain is a common condition in patients who are in intensive care units (ICU), precipitated and maintained by biological, psychological and social stimuli. Therefore, the management of patients in ICU with pain, requires an interdisciplinary approach to care which transcends the medical scope. The aims of this review were to present the strategies for assessment and management of pain in ICU patients, and to propose an interdisciplinary approach to deliver a more effective management in these units. The literature shows that the methods for pain assessment are diverse and that specific qualitative and quantitative measurements are used according to the patient's state of consciousness. The management of pain in the ICU is primarily determined by nursing and medical professionals despite the patients' psychological needs. Based on the evidence that pain management is multidisciplinary, it is proposed an interdisciplinary intervention which include the family.
\end{abstract}

Keywords: Pain; Intensive Care Units, pain measurement, behavior therapy, pain management.

1. Programa de Psicología, Universidad del Rosario. Bogotá, Colombia.

Correspondencia: Ximena Palacios-Espinosa, Dirección: Carrera 24 63C-69 Universidad del Rosario - Quinta de Mutis. Bogotá, Colombia. Correo electrónico: ximena.palacios@urosario.edu.co. Teléfono: 031-2970200 ext. 3486. 


\section{INTRODUCCIÓN}

De acuerdo con la Asociación Internacional para el Estudio del Dolor, este se define como una experiencia sensorial y emocional desagradable asociada con daño tisular, real o potencial, o descrita en los términos de dicho daño ${ }^{1}$. El dolor es un signo frecuente en las Unidades de Cuidado Intensivo (UCI ${ }^{2,3}$, y si bien es esperable, su mantenimiento no es lo ideal, ni por ello merece menos atención que las condiciones que han puesto en riesgo la vida del paciente, determinando su ingreso a estas Unidades.

Algunas enfermedades agudas cursan con dolor y suelen desencadenar el ingreso del paciente a la UCI; entre ellas, el infarto agudo de miocardio y otros problemas cardiacos $^{4,5}$, la pancreatitis ${ }^{4,6}$, las enfermedades vasculares ${ }^{4}$, la úlcera gástrica perforada ${ }^{4}$, la embolia pulmonar ${ }^{4}$, la embolia arterial periférica ${ }^{4}$, las quemaduras ${ }^{4}$ (en las que el dolor es el resultado de la lesión ${ }^{7}$ de los procedimientos, de los cuidados rutinarios de las zonas lesionadas ${ }^{7,8}$ o de infecciones de la herida ${ }^{8}$ ), y el trauma ${ }^{4}$ (específicamente el trauma cráneoencefálico ${ }^{9}$ ). Igualmente, algunas enfermedades crónicas cursan con algún tipo de dolor y pueden generar ingreso a la UCI; es el caso de la lumbalgia, las neuropatías, la fibromialgia y otros dolores musculares, las osteoartropatías, el cáncer y la cefalea ${ }^{4}$.

Aun más, en el periodo posoperatorio en UCI de cirugías abdominales o torácicas se presenta, además de las potenciales complicaciones pulmonares ${ }^{10}$ (especialmente tras intervención cardiaca ${ }^{11}$ ), dolor agudo $^{12}$ asociado también a otros eventos como neumonía bacteriana ${ }^{13}$.

Los procedimientos propios del cuidado del paciente en UCI, generan dolor ${ }^{14}$; específicamente, el cuidado de heridas, el retiro de sistemas de drenaje, la aspiración traqueal, la extracción de la vaina femoral, la introducción de un catéter central ${ }^{15}$, la broncoscopia, la traqueostomía percutánea por dilatación, la colocación del tubo torácico, el cateterismo venoso central, el cateterismo de arteria pulmonar ${ }^{16}$, el cambio de apósitos, la posición en los colchones de aire ${ }^{17}$, el cambio de posición ${ }^{18}$, los procedimientos repetidos, la fisioterapia ${ }^{19}$, las inyecciones y la aplicación de medicamentos por vía intravenosa (método IV push) ${ }^{20}$, las sondas nasogástricas, los tubos endotraqueales y las líneas centrales y arteriales ${ }^{21}$.

La ausencia de un tratamiento efectivo para el dolor, durante la estancia del paciente en la UCI, puede constituirse en un factor de riesgo para el desarrollo posterior de dolor crónico ${ }^{15}$. En cualquier caso, el dolor experimentado en una UCI es por sí mismo un factor estresante $^{21}$. En la UCI, predomina la experiencia del dolor agudo, el cual es fácilmente identificable por parte de los pacientes durante los dos primeros días de su estancia en esta Unidad ${ }^{22}$. Sin embargo, este puede potencialmente cronificarse ${ }^{23}$. Como se mencionó previamente, el control inadecuado del dolor agudo es uno de los principales predictores de la cronicidad ${ }^{24}$, así como el afecto negativo que la experiencia dolorosa pueda generar en el paciente crítico $^{25}$ pues en dichos casos, este puede inducir o magnificar la hipervigilancia hacia dolor y la activación fisiológica, generando una hipersensibilización ante el dolor ${ }^{25}$.

Lo anterior pone en evidencia que encontrarse hospitalizado en una UCI resulta ser un evento doloroso en el sentido más amplio del término. Así pues, uno de los grandes retos del equipo de salud de una UCI es lograr un manejo efectivo del dolor, lo que en la actualidad es considerado como estándar de calidad en el cuidado del paciente crítico y un gran reto para los intensivistas, pues se reconoce que el control inadecuado del dolor puede tener efectos nocivos y graves en diversos sistemas del paciente ${ }^{26}$. La literatura muestra que el trabajo interdisciplinario puede ser particularmente útil para lograr el manejo efectivo del dolor ${ }^{27}$. Si bien la evaluación de este signo se dificulta debido a los estados alterados de consciencia y a la intubación, que con frecuencia presentan estos pacientes ${ }^{28}$, esto no debe constituirse en una barrera ni en un factor para justificar la indiferencia ante el dolor del paciente. Al contrario, es necesario pensar en estrategias efectivas para su identificación y control. De acuerdo con lo anterior, este artículo tiene como objetivos presentar las estrategias de evaluación e intervención del dolor en pacientes que se encuentran en UCI y proponer una estrategia de abordaje interdisciplinario del dolor en estas unidades.

\section{La evaluación del dolor del paciente en UCI}

Aún con las limitaciones que supone la comunicación con el paciente en UCI, diversos métodos permiten obtener información sobre el dolor que puede estar experimentando. Barr, et al., recomiendan que la evaluación del dolor en UCI adultos se haga rutinariamente a todos los pacientes ${ }^{29}$.

Si bien la evaluación de la intensidad del dolor está siempre influida por la subjetividad de la experiencia dolorosa y por las características propias de la enfermedad, en la UCI ésta además pueda estar influida por los procedimientos dolorosos que se llevan a cabo $^{30}$. Los métodos subjetivos o de autorreporte 
son ampliamente utilizados ya que el paciente es considerado como la fuente de información más confiable $^{31}$. En pacientes que pueden emitir respuestas verbales, los métodos de evaluación del dolor son variados y se centran, principalmente, en la evaluación de su intensidad ${ }^{32}$. La escala de clasificación numérica es el instrumento más utilizado para evaluar el dolor en este tipo de pacientes ${ }^{33}$. Otros instrumentos disponibles son: la Escala Visual Analógica (EVA), y la Escala Descriptiva Verbal (Adjective Rating Scale, ARS) ${ }^{34}$, las cuales han sido descritas como medidas del dolor válidas y confiables en pacientes comunicativos verbales en la $\mathrm{UCI}^{35}$. Su uso puede extenderse a pacientes no verbales que puedan asentir con la cabeza, indicar palabras ${ }^{15} \mathrm{o}$ parpadear para señalar la severidad de su dolor cuando el profesional va anunciando descriptores de dolor o números que señalan su intensidad y cualidad ${ }^{33}$.

Además de la intensidad, otros instrumentos subjetivos permiten evaluar la cualidad del dolor; un ejemplo de ellos es el cuestionario de dolor de McGill, aunque hay que tener en cuenta las dificultades relacionadas con la validez del mismo en sus diversas traducciones ${ }^{36,37}$. Sin embargo, en el contexto específico de la UCI, se han hecho propuestas como la publicada por Granja, et al., quienes elaboraron un cuestionario de 14 preguntas para pacientes verbales en el que se evalúa, además del dolor, recuerdos de la estancia en la UCI, relaciones con los profesionales de la salud, sueños, pesadillas, trastornos del sueño, fatiga, dificultades para concentrarse y recordar eventos recientes ${ }^{38}$. No obstante, el uso de este último instrumento, aun no se ha extendido masivamente a la práctica clínica.

De otro lado, múltiples factores afectan el uso y desarrollo de métodos subjetivos para la evaluación del dolor en la UCI (dificultad para verbalizar como consecuencia de la intubación, barreras culturales y del lenguaje, y niveles alterados de consciencia) ${ }^{39}$. En consecuencia, los métodos objetivos del dolor se utilizan cuando los reportes que el paciente ofrece no son suficientes. Diversos instrumentos han permitido evaluar la presencia de dolor en pacientes no comunicativos de las UCI, entre ellos: (1) Pain Assessment Intervention and Notation (PAIN) Algorithm; (2) Nonverbal Pain Assessment Tool (NPAT); (3) Adult Nonverbal Pain Scale (NVPS); (4) Behavioral Pain Scale (BPS) $)^{40,41}$, diseñado para evaluar el dolor en pacientes inconscientes ventilados mecánicamente y/o sedados; (5) CriticalCare Pain Observational Tool (CPOT) $)^{40,42}$; (6) escala de Campbell; y, (7) Behavioral Pain Assessment Scale. Un reciente estudio realizado por Chanques, et al. con 30 pacientes hospitalizados en UCI y sedados, en delirium o bajo ventilación mecánica, buscó comprar las propiedades psicométricas del BPS, CPOT y NVPS, encontrando que las dos primeras tienen propiedades psicométricas similares en pacientes críticos intubados y no intubados ${ }^{43}$. Respecto de los pacientes ventilados mecánicamente, Payen, et al. resaltan que una evaluación sistemática del dolor puede considerarse como un criterio de buena práctica clínica en la UCI y que incluso, puede disminuir la dosis de opioides administrados al paciente crítico ventilado, la duración de la ventilación y la estancia en $\mathrm{UCI}^{18}$.

Estudios como el de Wøien, et al., han probado otras técnicas útiles para la evaluación del dolor que, si bien son poco utilizados, han demostrado ser útiles para enfocar el quehacer del personal de enfermería sobre los signos y síntomas asociados con la sedación y la analgesia de los pacientes en UCI, así como para reportar la frecuencia de dolor y de sedación, el número de días prescritos y la dosis de analgésicos y sedantes ${ }^{44}$.

A pesar de lo anterior, es claro que un paciente no comunicativo o con la capacidad verbal francamente disminuida, no debe ser la única fuente de información del dolor. De hecho, a través del informe sustituto o Surrogate Reporting, los familiares pueden ofrecer sus propias perspectivas acerca del dolor actual del paciente y proveer una descripción de los comportamientos que puedan indicar dolor $33,45,46$.

A su vez, es importante destacar que el personal de salud de la UCI utiliza indicadores fisiológicos para evaluar el dolor en pacientes críticos $^{33,47}$. Según Gélinas, et al., tras una exhaustiva revisión de archivos médicos de dos centros de salud que incluían información general y notas clínicas sobre el dolor del paciente, se logró identificar que los enfermeros prefieren documentar y evaluar comportamientos dolorosos (movimientos corporales, calidad del descanso, cumplimiento y asimilación del ventilador, medios de comunicación, entre otros) más que indicadores fisiológicos (respuestas cardiovasculares, respiratorias y cerebrales) ${ }^{48}$. Sobre esto, Barr, et al. recomiendan no utilizar solamente los signos vitales, ni aquellos instrumentos observacionales que los incluyan, para evaluar el dolor en estos pacientes. Indican que estos signos deben considerarse apenas como datos necesarios, pero no suficientes, para la evaluación del dolor ${ }^{29}$.

Por su parte, Mularski, et al. indican que es pertinente utilizar un listado de chequeo para la evaluación del dolor, y aplicarlo en las rondas que se realizan rutinariamente en las UCI. De esta manera, el paciente se sentirá más cómodo ${ }^{49}$. 
De acuerdo con lo mencionado en esta sección, es claro que actualmente hay disponibles métodos cualitativos y cuantitativos, psicológicos y fisiológicos que permitirían identificar la presencia del dolor en el paciente que ha ingresado en la UCI. No obstante, ¿cómo tratar efectivamente este síntoma una vez que se ha identificado?

\section{El tratamiento del dolor del paciente en UCI}

Es frecuente que el abordaje del dolor sea interdisciplinario y que esta estrategia se reporte como efectiva $^{50}$. Esta es una característica importante del equipo que trata el dolor, pero no por ello suficiente. Es necesario que el trabajo de varios profesionales se caracterice, entre otros, por ser coordinado y coherente ${ }^{51}$, para contribuir al alcance del objetivo común de establecer un diagnóstico correcto y estrategias de intervención más efectivas para el manejo y el control del dolor. En consecuencia, no basta con administrar analgésicos y sedantes, como se indica según la práctica médica común y los protocolos de manejo ${ }^{29}$, pues el dolor es una experiencia que amenaza la integridad física, psicológica y social del paciente en $\mathrm{UCI}^{52}$. De tal manera, es necesario admitir la multidimensionalidad del dolor y profundizar en el significado del mismo ${ }^{53}$.

En el escenario del cuidado intensivo la atención del dolor está centrada en el manejo dado por Medicina ${ }^{54}$ y Enfermería ${ }^{55,56}$, a pesar de que se reconocen las implicaciones psicológicas (ansiedad, depresión, sentimientos de desmoralización, trastornos del sueño, miedo) del dolor en pacientes ingresados en UCI, tanto a corto, como a largo plazo ${ }^{57}$. Al respecto, Celis-Rodriguez, et al., reportan que el manejo del dolor es un derecho del paciente e indican que entre los objetivos de la sedoanalgesia del paciente en UCI, se encuentran disminuir la ansiedad y lograr un nivel óptimo de comodidad $^{58}$. Sin embargo, estos autores no plantean la intervención de profesionales diferentes a médicos y enfermeros para resolver las necesidades del paciente con dolor en la UCI. Indican que pueden utilizarse estrategias no farmacológicas (masajes, relajación, musicoterapia, modificación de estímulos ambientales) en la intervención, pero ninguna de ellas alude a la intervención psicológica o por parte de otros profesionales ${ }^{58}$.

Las técnicas que se reconocen para el manejo del dolor en las UCI son básicamente farmacológicas ${ }^{58} \mathrm{e}$ incluyen la administración de opioides en diferentes modalidades por prescripción médica ${ }^{59}$, las técnicas multimodales para dar analgesia, las técnicas de analgesia regional periférica que incluyen bloqueos e infiltraciones, y la analgesia central regional con opioides ${ }^{59}$.
La intervención del paciente con dolor por parte del cuerpo médico y de enfermería, enfatiza en un abordaje farmacológico que incluye analgésicos y sedantes administrados de manera simultánea, tanto para la enfermedad como para los procedimientos dolorosos y molestos ${ }^{60,61}$. De acuerdo con Celis-Rodríguez, et al., "la sedación y la analgesia son parte integral en el manejo de los pacientes críticos en las UCI"58, y entre otros, tiene como objetivo el control del dolor.

A pesar de ello, cuando se utilizan de forma individual, los sedantes se administran con mayor frecuencia que los analgésicos. La razón de ello según Gélinas, et al., es que los profesionales buscan proveer comodidad a los pacientes y minimizar indicadores de ansiedad ${ }^{48}$. De hecho, esta es una de las razones que también justifican la sedoanalgesia de acuerdo con Celis-Rodríguez, et $a l^{58}$. Lo cierto es que el interés por el control del dolor en este contexto es evidente, así lo demuestran tanto la "Guía de práctica clínica basada en la evidencia para el manejo de la sedoanalgesia en el paciente adulto críticamente enfermo" 58 y el trabajo de revisión de las "Guías de Práctica Clínica para el uso sostenido de sedantes y analgésicos en adultos críticamente enfermos", realizado por Barr et al., en el cual se propone que dichas guías proporcionan una ruta de orientación basada en la evidencia para la construcción de protocolos dirigidos, entre otros, a la prevención y el tratamiento del dolor ${ }^{29}$.

Por su parte, Bender reporta que los analgésicos más utilizados en la UCI son los opioides y a menudo la lidocaína local. Además, se educa a los pacientes sobre el tratamiento planeado ${ }^{62}$.

Asimismo, estudios como el de Reader et al., han resaltado que médicos y enfermeros deben poder establecer una comunicación efectiva e interdisciplinaria para alcanzar el mejor cuidado de los pacientes críticos. De hecho, varios protocolos como el SPIKES (Setting; Perception; Invitation or information; Knowledge; Empathy; Summarize or strategize) dirigido a informar malas noticias a pacientes y familiares, creando un marco teórico de referencia para las discusiones difíciles; y otros métodos como el NURSE (Naming, Understanding, Respecting, Supporting, Exploring), que permite responder de manera empática a las emociones del paciente ${ }^{63}$, son ejemplos para lograr este tipo de comunicación. Básicamente y siguiendo la idea enunciada por Kaplan, se trata de instaurar una relación recíproca, empática, fundamentada en el respeto y la dignidad del paciente ${ }^{63}$. En el contexto de una UCI, comunicarse efectivamente con pacientes dependientes de la ventilación mecánica es esencial 
para que sus necesidades fisiológicas y psicológicas sean atendidas y el plan de atención se ajuste a ellas ${ }^{60}$. Así, un sistema de comunicación efectiva requiere tanto de la colaboración del equipo de salud como del paciente y de su familia ${ }^{65}$. En coherencia con lo anterior, Krimshtein, et al. concluyen que la "comunicación efectiva es un componente esencial del cuidado de alta calidad en la UCI"65.

Por ser el dolor una experiencia emocional y afectiva, además de sensorial ${ }^{53}$, en la intervención del mismo es pertinente considerar los aspectos psicológicos y su impacto en el paciente y en la familia ${ }^{52}$. De hecho, además del control de la ansiedad previamente mencionado, y en total acuerdo con Bender, la necesidad de reconocer, evitar y manejar los procedimientos dolorosos de la UCI no solo tiene implicaciones de tipo ético sino también es de fundamental importancia para el paciente y para su familia ${ }^{62}$. Por esto, se propone que la intervención psicológica del dolor en la UCI esté dirigida no solo al paciente sino a su familia y a los miembros del equipo de salud.

En lo referente al trabajo del psicólogo en el manejo del dolor en el paciente de la UCI, la literatura es francamente escasa. Sin embargo, la disciplina psicológica tiene un papel central no solo en el estudio sino en la evaluación y el tratamiento del dolor ${ }^{53}$. Por ejemplo, autores como Morley afirman que en el contexto clínico, la intervención de un psicólogo es pertinente cuando el reporte del paciente, sus emociones o su conducta frente al dolor, difieren francamente de la opinión médica al respecto. Además, indica que el dolor puede tener efectos profundos en la vida de una persona, mediados por condiciones de frustración, ansiedad, catastrofización, miedo, entre otras ${ }^{66}$.

En lo que respecta al paciente en UCI, este se encuentra en un ámbito estresante en el que característicamente se generan diversidad de experiencias, pensamientos, emociones y sentimientos negativos ${ }^{67}$. Entre las experiencias cuyo componente emocional es comúnmente negativo, está el dolor que experimentan estos pacientes que, en congruencia con Esper y Hernández, involucra variables psicológicas que influyen de manera significativa en la respuesta positiva o negativa de los pacientes al tratamiento médico ${ }^{68}$.

En particular, los factores psicológicos pueden considerarse como mediadores de la percepción, el mantenimiento y la exacerbación del dolor ${ }^{53}$.

Las distintas corrientes psicológicas comprenden y abordan el dolor de manera diversa; por ejemplo, el
Conductismo ha estudiado con especial interés la conducta dolorosa ${ }^{69}$, proponiendo modelos como el de Fordyce (1976) para la explicación de la conducta, e igualmente, favoreciendo la construcción de instrumentos de evaluación e intervención terapéutica específica del dolor ${ }^{70}$. Por otra parte, el Cognitivismo ha destacado la importancia del significado y la experiencia del dolor, observando la influencia de los componentes motivacionales y de pensamiento, y examinando las variables antecedentes, modificadoras y moduladoras, que incluyen las creencias, las atribuciones, las expectativas, la autoeficacia, el autocontrol, la atención, el afrontamiento, la resolución de problemas, las autoinstrucciones y la imaginación ${ }^{71}$.

Los factores psicológicos del dolor involucran principalmente dos dimensiones: la dimensión cognoscitiva, que refiere a las creencias, valores culturales y variables cognitivas, y la dimensión afectiva que implica la cualidad subjetiva y emocional de la experiencia de dolor ${ }^{72}$. En concreto, el componente afectivo incorpora toda una gama de emociones negativas como la depresión, el miedo y la ira, las cuales se han relacionado con el origen, el mantenimiento y la exacerbación del dolor (específicamente del dolor crónico $^{73}$ ), generando efectos adversos en la calidad de vida del paciente, en la capacidad funcional y en la respuesta al tratamiento farmacológico ${ }^{74}$. Asimismo, la falta de expresión emocional está relacionada con experiencias de mayor dolor ${ }^{75}$.

Anteriormente se ha mencionado que el paciente en UCI sufre de ansiedad y, por tanto, es importante resaltar la relación entre ansiedad y dolor por ser la primera una de las variables potenciadoras y perpetuadoras del dolor y, además, una de las respuestas psicológicas de la experiencia de dolor más identificadas. Gaviria, et al. han estudiado la influencia de las emociones negativas (miedo, tristeza, ira) en el dolor crónico, así como la morbilidad psicológica asociada con esta (ansiedad y depresión), encontrando que en algunos individuos el dolor antecede a las emociones negativas, mientras que en otros las emociones negativas preceden al dolor ${ }^{69}$. Tradicionalmente se ha dicho que el dolor agudo se asocia con la ansiedad y el dolor crónico con la depresión. En general, no hay resultados concluyentes sobre esta relación ${ }^{69}$. Ciertamente, y en acuerdo con Truyols, et al., la depresión es una condición comúnmente asociada con el dolor, y cuando está presente en pacientes con dolor crónico, incrementa las distorsiones cognoscitivas frente a la enfermedad e intensidad del dolor asociado a procedimientos en la UCI, así como la dificultad para expresar sentimientos negativos ${ }^{53}$. 
De acuerdo con lo anterior, es pertinente indicar que el psicólogo tendrá dentro de sus funciones en la UCI realizar evaluación e intervención psicológica de los aspectos afectivos y emocionales propios del proceso que la condición médica crítica del paciente implica, entre ellos el dolor, contribuyendo de esa manera al ajuste y la adaptación a la enfermedad y al contexto de la unidad.

De tal manera, el psicólogo deberá evaluar si el paciente en UCI presenta tanto ansiedad como depresión mediante diversas estrategias e instrumentos psicométricos, y a partir de los resultados obtenidos, deberá ocuparse del abordaje de los pensamientos distorsionados sobre su experiencia dolorosa ${ }^{53}$. Lo más importante sin duda, es que el psicólogo realice su intervención desde la evidencia y, por tanto, es recomendable que tenga como referencia alguno de los siguientes marcos teóricos: (1) El enfoque de autorregulación (técnicas de retroinformación biológica [biofeedback], relajación y respiración, hipnosis, terapia de autorregulación y mindfulness), (2) el enfoque comportamental (terapia de comportamiento operante y evitación del miedo), (3) la terapia cognoscitivo-comportamental y (4) la terapia de aceptación y compromiso, así como otras terapias conductuales de tercera generación ${ }^{76}$.

Intervención interdisciplinaria del dolor en UCI: una propuesta de la interacción entre Medicina Enfermería - Psicología

La colaboración interdisciplinaria es el proceso que se produce a nivel interpersonal a través del cual los miembros de diversas disciplinas contribuyen a un objetivo común ${ }^{77}$. La interdisciplinariedad es un tema de interés creciente en diferentes contextos, entre ellos las instituciones de salud. Este concepto lleva implícito el que expertos de diferentes disciplinas interactúen para buscar soluciones a problemas, donde la que la intervención de una sola disciplina resulta insuficiente. El trabajo interdisciplinario es dinámico, colaborativo, integrativo, vinculante, permite mezclar ideas y conceptos desde diferentes disciplinas y puede facilitar el trabajo en equipo y su eficiencia ${ }^{51}$.

El problema a resolver en este caso específico es el dolor experimentado por el paciente en la UCI que, al ser una experiencia compleja con componentes sensoriales y psicológicos, puede ser resuelto de una manera más eficaz a través del trabajo colaborativo. Además, es fundamental comprender que el dolor no afecta exclusivamente al paciente en UCI, sino también a su familia ${ }^{78}$, específicamente al cuidador, que observa y reconoce la conducta dolorosa en su pariente y que, en consecuencia, puede contribuir al informar al equipo sobre el dolor del paciente. Diversos estudios explican que, al igual que el paciente, la familia presenta niveles de ansiedad y depresión elevados durante toda la estancia del paciente en la UCI, relacionados con la evolución del proceso y con la falta de contacto con su ser querido ${ }^{79}$, lo que puede incrementar la posibilidad de que no atiendan sus propias necesidades. Además, la estancia del paciente en la UCI puede producir que la familia vivencie una serie de alteraciones psicológicas y sociales como estrés, desorganización y sentimientos de desamparo ${ }^{80}$, por lo que deben utilizar estrategias de afrontamiento que les permitan adaptarse a esta nueva situación.

Es fundamental dotar a los familiares del paciente en UCI de sensación de control. Presenciar el dolor de un ser querido genera altos niveles de estrés y aún más si se trata de una persona cuyo estado de salud es crítico. Por tanto, la propuesta es que la familia sea entrenada para identificar los signos de dolor en su pariente y que, a la vez, sea una fuente de comunicación directa con el paciente y con el equipo de salud. Además del vínculo que los une al paciente, y por tanto del conocimiento que tienen del mismo, si se les entrena de una manera técnica y específica, los familiares del paciente pueden aprender a identificar con precisión los signos de dolor y reportarlos oportunamente a los miembros del equipo de salud para una intervención efectiva (Figura 1).

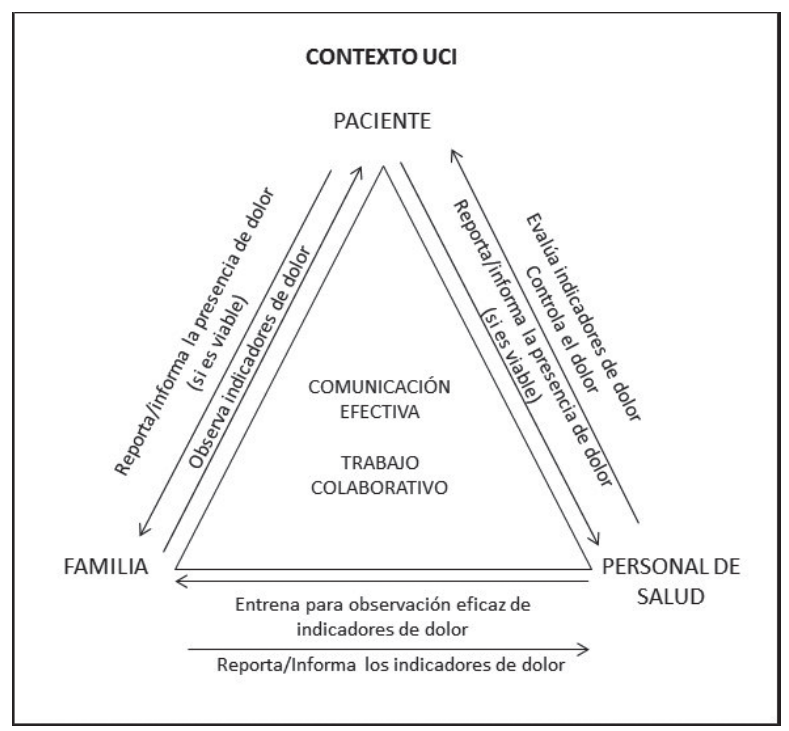

Figura 1. Dinámica característica de la interacción entre paciente-familia y personal de salud en el trabajo interdisciplinario para el manejo del paciente con dolor en el contexto de una UCI.

La propuesta, centrada en que el manejo del dolor es un derecho del paciente ${ }^{58}$, es que la intervención 
interdisciplinaria inicie con la evaluación del dolor y como en cualquier otro proceso terapéutico, la evaluación sea continua. Es decir, que desde el ingreso del paciente a la UCI habrá dos objetivos transversales para lograr el trabajo interdisciplinario y permitir un óptimo manejo del dolor: (1) evaluar la presencia del dolor durante toda la estancia en la Unidad a través de instrumentos válidos y confiables, así como evaluar la efectividad del tratamiento analgésico y de intervenciones no farmacológicas basados en la evidencia, antes, durante y después de su administración; (2) garantizar que todos los miembros del equipo de salud de la UCI dominan habilidades de comunicación efectiva que les permitan ser multiplicadores de las mismas, no solo entre sus colegas, sino con la familia del paciente que ha ingresado a la UCI. La comunicación efectiva es un elemento fundamental para establecer relaciones interpersonales funcionales entre paciente-familiamiembros del equipo de salud.

Médicos, enfermeros y psicólogos estarán capacitados para evaluar y tratar el dolor, con competencias y niveles de análisis diferentes, de acuerdo con la perspectiva propia de cada profesión (farmacológica, centrada en el cuidado o centrada en la conducta) que, al ser compartida, permite el abordaje interdisciplinario del dolor del paciente en UCI. La estrategia consiste en que cada profesional desde su campo de acción, esté en capacidad de proporcionar información sobre el dolor, para así complementar el conocimiento que tienen los demás miembros del equipo de salud sobre el tema. Se trata de construir un espacio para el conocimiento en el que se respeten y se reconozcan los alcances y los límites de las diferentes profesiones, rescatando y comprendiendo las funciones de cada uno en el manejo interdisciplinario del paciente con dolor dentro de la UCI. Cada uno de los miembros del equipo se constituye como una fuente activa, propositiva y sistemática, de apoyo para el paciente y la familia y, por consiguiente, será fundamental que articulen esfuerzos para hacer de la estancia en UCI, la mejor posible (Figura 1).

Aun mas, para lograr un trabajo interdisciplinario, es condición que todos los miembros del equipo estén completamente educados respecto al manejo del dolor. Se ha reportado que algunas creencias actúan como barreras tanto para el paciente como para el profesional de la salud en la reducción y control del dolor en la UCI, como por ejemplo, que el paciente esté sedado, los deseos de la familia y las políticas del hospital ${ }^{81,82}$. Además, el conocimiento del personal de salud acerca de los procedimientos y enfermedades que producen dolor en la unidad y sus actitudes hacia el mismo, son componentes importantes que determinan sus comportamientos en relación con el manejo del dolor ${ }^{84}$. Sin embargo, y a pesar de que ellos tienen un rol importante en el cuidado del dolor del paciente, varios estudios han demostrado que la información que manejan al respecto es inapropiada ${ }^{32,82}$, en específico en relación con los riesgos de la sedación, la depresión respiratoria y/o el poder adictivo de los opioides, lo que a menudo conduce a la administración inadecuada de medicamentos ${ }^{32}$. Las intervenciones basadas en la educación, como el uso de programas estructurados que proveen información acerca del dolor y los recursos y servicios disponibles para su evaluación y tratamiento adecuado ${ }^{83}$, son una estrategia válida para mejorar el cuidado de los pacientes y sus respuestas al dolor, pues de acuerdo con Patiraki, et al., pueden mejorar el conocimiento del personal médico y sus actitudes hacia el dolor ${ }^{84}$.

\section{CONCLUSIONES}

El dolor es un factor de posibles complicaciones para el paciente en $\mathrm{UCI}^{85}$. Como todos los pacientes, quienes se encuentran ingresados en estas unidades tienen derecho a recibir tratamiento para el dolor. La literatura sobre el manejo del dolor del paciente en UCI reconoce que el tratamiento de este signo debe hacerse de manera multidisciplinaria y que esta medida puede optimizar los resultados ${ }^{85}$. Sin embargo, no se encontró literatura que respalde la atención interdisciplinaria del dolor; es posible que el concepto de multidisciplinariedad sea utilizado de manera indiferenciada con interdisciplinariedad. Igualmente, se enfatiza en que médicos y enfermeros son los profesionales encargados de controlar el dolor.

El manejo del dolor en el paciente crítico se centra en medidas farmacológicas, desconociendo aquellas no farmacológicas como las que pueden ser suministradas por los psicólogos, profesionales que serían parte fundamental del equipo si se considera la complejidad del dolor y el componente afectivo y emocional que lo caracteriza.

La importancia de todas las modalidades de intervención es crítica en un contexto en el que con una alta probabilidad aparecerá el dolor porque los procedimientos en sí mismos son dolorosos, porque las consecuencias de la enfermedad son dolorosas o porque la enfermedad de base es dolorosa. Por tanto, si bien la analgesia se convierte en una prioridad, el dolor es una experiencia que trasciende el aspecto sensorial, por lo que requiere de un abordaje interdisciplinario e integral en el que las estrategias de intervención sobrepasen el 
aspecto farmacológico, atendiéndose las necesidades psicológicas y sociales del paciente y de su familia.

Esta última, es un elemento esencial en la atención integral al paciente en tanto se constituye como fuente de información sobre el dolor, pero también en fuente de bienestar para el paciente, quien siente su respaldo y su contención.

La propuesta específica que se deriva de esta revisión de tema, es que la intervención integral del paciente con dolor en UCI debe incluir tres actores fundamentales: el paciente, la familia y el personal de salud. La condición para este tipo de intervención es la comunicación efectiva y el resultado esperado es común para los tres: controlar el dolor, contribuir a disminuir el sufrimiento y aumentar las probabilidades de que el paciente reciba atención integral, brindándose la mayor garantía posible de que sus necesidades frente al dolor sean satisfechas.

\section{REFERENCIAS}

1. Merskey J, Albe-Fessard DG, Bonica JJ, Carmon A, Dubner R, Kerr FWL, et al. Pain terms: a list with definitions and notes on usage. Recommended by the IASP subcommittee on taxonomy. Pain. 1979; 6(3): 249-252.

2. Stein-Parbury J, McKinley S. Patients' experiences of being in an intensive care unit: a select literature review. Am J Crit Care. 2000; 9(1): 20-27.

3. Choi J, Hoffman LA, Schulz R, Tate JA, Donahoe MP, Ren D, et al. Self-reported physical symptoms in intensive care unit (ICU) survivors: Pilot exploration over four months post-ICU discharge. J Pain Symptom Manage. 2014; 47(2): 257-270. DOI: 10.1016/j.jpainsymman.2013.03.019.

4. Whizar-Lugo VM, Flores-Carrillo JC, MarínSalazar G, Benavides-Vázquez A. Diagnóstico y tratamiento del dolor agudo en el paciente crítico. Anest Mex. 2008; 20(2): 85-90.

5. Suárez JC, Rodríguez L, Enríquez CL, Colombié R. Comportamiento del infarto agudo de miocardio en la unidad de cuidados intensivos. Rev Cubana Med. 2002; 41(1): 25-28.

6. Torres NM, Acuña EG, Sánchez R, Pena D, Mota R. Pancreatitis crónica secundaria a litiasis primaria del conducto de wirsung, clínica y tratamiento quirúrgico. Caso clínico. Informed. 2010; 12(4): 201-206.

7. Abdi S, Zhou Y. Management of pain after burn injury. Curr Opin Anaesthesiol. 2002; 15(5): 563567. DOI: http://dx.doi.org/10.1097/00001503200210000-00015.
8. Tengvall OM, Björnhagen VC, Lindholm C, Jonsson CE, Wengström Y. Differences in pain patterns for infected and noninfected patients with burn injuries. Pain Manag Nurs. 2006; 7(4): 176-182. DOI: http:// dx.doi.org/10.1016/j.pmn.2006.09.002.

9. Stanik-Hutt JA, Soeken KL, Belcher AE, Fontaine DK, Gift AG. Pain experiences of traumatically injured patients in a critical care setting. Am J Crit Care. 2001; 10(4): 252-259.

10. Do Nascimento P, Módolo N, Andrade S, Guimarães M, Braz LG, El Dib R. Incentive spirometry for prevention of postoperative pulmonary complications in upper abdominal surgery. Cochrane Database Syst Rev. 2014; 2: 1-43. DOI: http:// dx.doi.org/10.1002/14651858.CD006058.pub3 .

11. Wynne R, Botti M. Postoperative pulmonary dysfunction in adults after cardiac surgery with cardiopulmonary bypass: clinical significance and implications for practice. Am J Crit Care. 2004; 13(5): 384-393.

12. Milgrom LB, Brooks JA, Qi R, Bunnell K, Wuestefeld S, Beckman D. Pain levels experienced with activities after cardiac surgery. Am J Crit Care. 2004; 13(2): 116-125.

13. Bongard FS, Sue DY. Diagnóstico y tratamiento en cuidados intensivos. 2a ed. México D.F.: Editorial Manual Moderno; 2003. p 113-138.

14. Siffleet J. Young J, Nikoletti S, Shaw T. Patients' self-report of procedural pain in the intensive care unit. J Clin Nurs. 2007; 16(11): 2142-2148. DOI: 10.1111/j.1365-2702.2006.01840.x.

15. Stolic S, Mitchell ML. Pain management for patients in cardiac surgical intensive care units has not improved over time. Aust Crit Care. 2010; 23(3): 157-159. DOI: http://dx.doi.org/10.1016/j. aucc.2010.04.004.

16. Phua GC, Wahidi MM. ICU procedures of the critically ill. Respirology. 2009; 14(8): 1092-1097. DOI: 10.1111/j.1440-1843.2009.01643.x.

17. Aslan FE, Badir A, Arli SK, Cakmkci H. Patients' experience of pain after cardiac surgery. Contemp Nurse. 2009-10; 34(1): 48-54. DOI: http://dx.doi. org/10.5172/conu.2009.34.1.048.

18. Payen JF, Bosson JL, Chanques G, Mantz J, Labarere $\mathrm{J}$, DOLOREA Investigators. Pain assessment is associated with decreased duration of mechanical ventilation in the intensive care unit: a post hoc analysis of the DOLOREA study. Anesthhesiol. 2009; 111(6): 1308-1316. DOI: http://dx.doi. org/10.1097/ALN.0b013e3181c0d4f0.

19. Rojas JG. Cuidado de enfermería al paciente quemado en estado crítico de salud. En: Pulgarín AM, Osorio SP, Varela LE, eds. Cuidado del paciente 
Intervención interdisciplinaria del paciente con dolor en Unidades de Cuidado Intensivo (UCI): Revisión de tema

en estado crítico. Medellín, Colombia: Corporación para investigaciones biológicas; 2012. p. 293-312.

20. Kline WH, Turnbull A, Labruna VE, Haufler L, DeVivio S, Ciminera P. Enhancing pain management in the PICU by teaching guided mental imagery: a quality-improvement project. J Pediatr Psychol. 2010; 35(1): 25-31. DOI: http://dx.doi.org/10.1093/ jpepsy/jsp030.

21. Aïssaoui Y, Zeggwagh AA, Zekraoui A, Abidi $\mathrm{K}$, Abougal R. Validation of a behavioral pain scale in critically ill, sedated, and mechanically ventilated patients. Anesth Analg. 2005; 101(5): 1470-1476. DOI: http://dx.doi.org/10.1213/01. ANE.0000182331.68722.FF.

22. Bertolini G, Minelli C, Latronico N, Cattaneo A, Mura G, Melotti RM, et al. The use of analgesic drugs in postoperative patients: the neglected problem of pain control in intensive care units. An observational, prospective, multicenter study in 128 Italian intensive care units. Eur J Clin Pharmacol. 2002; 58(1): 73-77. DOI: http://dx.doi.org/10.1007/ s00228-002-0432-y.

23. Martí JL. Tratamiento del dolor postoperatorio en UCI. En: Primer simposio virtual de dolor, medicina paliativa y avances en farmacología del dolor. Buenos Aires: Asociación Argentina para el Estudio del Dolor; 2001.

24. Diallo B, Kautz DD. Better pain management for elders in the intensive care unit. Dimens Crit Care Nurs. 2014; 33(6): 316-319. DOI: 10.1097/ DCC. 0000000000000074.

25. Janssen SA. Negative affect and sensitization to pain. Scand J Psychol. 2002; 43(2): 131-137. DOI: http://dx.doi.org/10.1111/1467-9450.00278.

26. Ehieli E, Yalamuri S, Brudney CS, Pyati D. Analgesia in the surgical intensive care unit. Postgrad Med J. 2017; 93(1095): 38-45. DOI: http:// dx.doi.org/10.1136/postgradmedj-2016-134047.

27. Pietilä Holmner E, Fahlström M, Nordström A. The effects of interdisciplinary team assessment and a rehabilitation program for patients with chronic pain. Am J Phys Med Rehabil. 2013; 92(1): 77-83. DOI: 10.1097/PHM.0b013e318278b28e.

28. Varndell W, Fry M, Elliott D. A systematic review of observational pain assessment instruments for use with nonverval intubated critically ill adult patients in emergency department: an assessment of their suitability and psychometric properties. J Clin Nurs. 2017; 26(1-2): 7-32. DOI: 10.1111/jocn.13594.

29. Barr J, Fraser GL, Puntillo K, Wesley E, Gélinas C, Dasta JF, et al. Clinical practice guidelines for the management of pain, agitation, and delirium in adult patients in the intensive care unit. Crit
Care Med. 2013; 41(1): 263-306. DOI: 10.1097/ CCM.0b013e3182783b72.

30. Puntillo K, Nelson JE, Weissman D, Cutris R, Weiss $\mathrm{S}$, Frontera J, et al. Palliative care in the ICU: relief of pain, dyspnea, and thirst --a report from the IPALICU Advisory Board. Intensive Care Med. 2014; 40: 235-248. DOI: 10.1007/s00134-013-3153-z.

31. Haslam L, Dale C, Knechtel L, Rose L. Pain descriptors for critically ill patients unable to selfreport. J Adv Nurs. 2012; 68(5): 1082-1089. DOI: 10.1111/j.1365-2648.2011.05813.x.

32. Marino PL. The ICU Book. 4a ed. Philadelphia (PA): Wolters Kluwer Health/Lippincott Williams \& Wilkins; 2014. p 901-918.

33. Puntillo KA, Pasero C, Li D, Mularski RA, Grap MJ, Erstad BL, et al. Evaluation of pain in ICU patients. Chest. 2009; 135(4): 1069-1074. DOI: 10.1378/chest.08-2369.

34. Chanques G, Viel E, Constantin JM, Jung B, de Lattre S, Carr J, et al. The measurement of pain in intensive care unit: comparison of 5 self-report intensity scales. Pain. 2010; 151(3): 711-721. DOI: 10.1016/j.pain.2010.08.039.

35. Vásquez -Ortega KC, García-Ramírez R, CruzRamírez M, Vásquez-Morales A. Electroterapia como manejo alternativo de dolor en quemaduras. Rev Sanid Milit Mex. 2008; 62(3): 141-144.

36. Boyle GJ, Fernández E, Ortet G. El cuestionario de dolor de McGill (McGill Pain QuestionnaireMPQ): Consideraciones lingüísticas y estadísticas. Rev Psicol. 2003; 12(1): 111-119.

37. Arroyo-Novoa CM, Figueroa-Ramos MI, Puntillo KA, Stanik-Hutt J, Thompson CL, White C, et al. Pain related to tracheal suctioning in awake acutely and critically ill adults: a descriptive study. Intensive Crit Care Nurs. 2008; 24(1): 20-27. DOI: 10.1016/j. iccn.2007.05.002.

38. Granja C, Lopes A, Moreira S, Dias C, CostaPereira A, Carneiro A, et al. Patients' recollections of experiences in the intensive care unit may affect their quality of life. Crit Care. 2005; 9(2): R96-R109. DOI: $10.1186 / \mathrm{cc} 3026$.

39. Puntillo KA, Morris AB, Thompson CL, StanikHutt J, White CA, Wild LR. Pain behaviors observed during six common procedures: results from thunder project II. Crit Care Med. 2004; 32(2): 421-427. DOI: 10.1097/01.CCM.0000108875.35298.D2.

40. Rijkenberg S, Stilma W, Endeman H, Bosman RJ, Oudemans-van Straaten HM. Pain measurement in mechanically ventilated critically ill patients: behavioral pain scale versus critical-care pain observation tool. J Crit Care. 2015; 30(1): 167-172. DOI: $10.1016 /$ j.jcrc.2014.09.007. 
41. Payen JF, Bru O, Bosson JL, Lagrasta A, Novel E, Deschaux I, et al. Assessing pain in critically ill sedated patients by using a behavioural pain scale. Crit Care Med. 2001; 29(12): 2258-2263. DOI: 10.1097/00003246-200112000-00004.

42. Li Q, Wan X, Gu C, Yu Y, Huang W, Li S, et al. Pain assessment using the critical-care pain observation tool in chinese critically ill ventilated adults. J Pain Symptom Manage. 2014; 48(5): 975-982. DOI: 10.1016/j.jpainsymman.2014.01.014.

43. Chanques G, Pohlman A, Kress JP, Molinari N, de Jong A, Jaber S, et al. Psychometric comparison of three behavioural scales for the assessment of pain in critically ill patients unable to self-report. Crit Care. 2014; 18(5): R160. DOI: 10.1186/cc14000.

44. Wøien H, Værøy H, Aamodt G, Bjørk IT. Improving the systematic approach to pain and sedation management in the ICU by using assessment tools. J Clin Nurs. 2012; 23(11-12): 1552-1561. DOI: 10.1111/j.1365-2702.2012.04309.x.

45. Herr K, Coyne PJ, Key T, Manworren R, McCaffery $\mathrm{M}$, Merkel S, et al. Pain assessment in the nonverbal patient: position statement with clinical practice recommendations. Pain Manag Nurs. 2006; 7(2): 44-52. DOI: http://dx.doi.org/10.1016/j. pmn.2006.02.003.

46. Desbiens NA, Mueller-Rizner N. How well do surrogates assess the pain of seriously ill patients?. Crit Care Med. 2000; 28(5): 1347-1352.

47. Giraldo IC, Crespo LJ. Cuidado de enfermería durante el transporte interhospitalario del paciente en estado crítico. En: Pulgarín AM, Osorio SP, Londoño LE, eds. Cuidado del paciente en estado crítico. Medellín, Colombia: Corporación para investigaciones biológicas; 2012. p. 369-392.

48. Gélinas C, Fortier M, Viens C, Fillion L, Puntillo $\mathrm{K}$. Pain assessment and management in critically ill intubated patients: a retrospective study. Am J Crit Care. 2004; 13(2): 126-136.

49. Mularski RA, Osborne ML. Palliative care and intensive care unit care: daily intensive care unit care plan checklist \#123. J Palliat Med. 2006, 9(5): 1205-1206. DOI: 10.1089/jpm.2006.9.1205.

50. Dysvik E1, Natvig GK, Eikeland OJ, Lindstrøm TC. Coping with chronic pain. Int J Nurs Stud. 2005; 42(3): 297-305. DOI: 10.1016/j. ijnurstu.2004.06.009.

51. Choi BC, Park AW. Multidisciplinarity, interdisciplinarity and transdisciplinarity in health research, services, education and policy: 1 . Definitions, objectives, and evidence of effectiveness. Clin Invest Med. 2006. 29(6): 351-364.

52. Failde I. El dolor crónico, algo más que un problema de quien lo padece. Rev Soc Esp Dolor. 2014; 21(1): 1-2.

53. Truyols Taberner MM, Pérez Pareja J, Medinas Amorós MM, Palmer Pol A, Sesé Abad A. Aspectos psicológicos relevantes en el estudio y el tratamiento del dolor crónico. Clín Salud. 2008, 19(3): 295-320.

54. Azoulay E, Pochard F. Communication with family members of patients dying in the intensive care unit. Curr Opin Crit Care. 2003; 9(6): 545-550.

55. Davidson JE, Winkelman C, Gélinas C, Dermenchyan A. Pain, agitation, and delirium guidelines: nurses' involvement in development and implementation. Crit Care Nurse. 2015; 35(3): 1732. DOI: $10.4037 / \operatorname{ccn} 2015824$.

56. Gélinas C, Arbour C, Michaud C, Robar L, Côte J. Patients and ICU nurses' perspectives of non-pharmacological interventions for pain management. Nurs Crit Care. 2012; 18(6): 307-318. DOI: $10.1111 /$ j.1478-5153.2012.00531.x.

57. Sigakis MJG, Bittner EA. Ten myths and misconceptions regarding pain management in the ICU. Crit Care Med. 2015; 43(11): 2468-2478. DOI: 10.1097/CCM.0000000000001256.

58. Celis-Rodríguez E, Birchenall C, de la Cal MÁ, Castorena Arellano G, Hernández A, Ceraso D, et al. Guía de práctica clínica basada en la evidencia para el manejo de la sedoanalgesia en el paciente adulto críticamente enfermo. Med Intensiva. 2013; 37(8): 519-574. DOI: 10.1016/j.medin.2013.04.001.

59. American Society for Anesthesiologists Task Force on acute pain management. Practice guidelines for acute pain management in the perioperative setting: an updated report. Anesthesiol. 2012; 2(116): 248273. DOI:10.1097/ALN.0b013e31823c1030.

60. Jerath A, Ferguson ND, Steel A, Wijeysundera D, Macdonald J, Wasowicz M. The use of volatile anesthetic agents for long-term critical care sedation (VALTS): study protocol for a pilot randomized controlled trial. Trials. 2015; 16: 560. DOI: 10.1186/ s13063-015-1083-5.

61. Kunisawa T, Fujimoto K, Kurosawa A, Nagashima M, Matsui K, Hayashi D, et al. The dexmedetomidine concentration required after remifentanil anesthesia is three-fold higher than that after fentanyl anesthesia or that for general sedation in the ICU. Ther Clin Risk Manag. 2014; 10: 797-806. DOI: https://doi. org/10.2147/TCRM.S67211.

62. Bender BG. Pain control in the intensive care unit: new insight into an old problem. Am J Respir Crit Care Med. 2014; 189(1): 9-10. DOI: 10.1164/ rccm.201311-2059ED.

63. Kaplan M. SPIKES: a framework for breaking bad news to patients with cancer. Clin J Oncol 
Intervención interdisciplinaria del paciente con dolor en Unidades de Cuidado Intensivo (UCI): Revisión de tema

Nurs. 2010; 14(4): 514-516. DOI: 10.1188/10. CJON.514-516.

64. Grossbach I, Stranberg S, Chlan L. Promoting effective communication for patients receiving mechanical ventilation. Crit Care Nurse. 2011; 31(3): 46-60. DOI: 10.4037/ccn2010728.

65. Krimshtein NS, Luhrs CA, Puntillo KA, Cortez TB, Livote EE, Penrod JD, et al. Training nurses for interdisciplinary communication with families in the intensive care unit: an intervention. J Palliat Med. 2011; 14(12): 1325-1332. DOI: 10.1089/ jpm.2011.0225.

66. Morley S. Psychology of pain. B J Anaesth. 2008; 101(1): 25-31. DOI: 10.1093/bja/aen123.

67. Contreras Moreno AM, Palacios-Espinosa $\mathrm{X}$. Contribuciones de la Psicología al manejo interdisciplinario del paciente en Unidad de Cuidados Intensivos (UCI). Rev Univ Ind Santander Salud 2014; 46 (1): 47-60

68. Esper RC, Hernández K. La importancia de la psicología de enlace en la unidad de terapia intensiva. Rev Invest Med Sur Mex. 2011; 18(3): 118-127.

69. Gaviria AM, Vieco PL. Aspectos psicológicos del dolor crónico. En: Cadavid AM, Estupiñán JR, Vargas JJ, eds. Dolor y cuidados paliativos. Medellín, Colombia: Corporación para investigaciones biológicas; 2005. p. 32-36.

70. Fordyce WE. Behavioral methods for chronic pain and illness. St. Louis (MO): Mosby; 1976. p. 1-17.

71. Turk DC, Meichenbaum D, Genest M. Pain and behavioral medicine: a cognitive-behavioral perspective. New York (NY): Guilford Press; 1983. p. 73-118.

72. Melzack R, Casey KL. Sensory, motivational, and central control determinants of pain. A new conceptual model. In: Kenshalo D, ed. The skin senses. Springfield (MA): Charles C. Thomas; 1968. p. 423-443.

73. Ackerman MD, Stevens MJ. Acute and chronic pain: pain dimensions and psychological status. J Clin Psychol. 1989; 45(2): 223-228. DOI: 10.1002/1097-4679(198903)45:2<223::AIDJCLP2270450208>3.0.CO;2-Y.

74. Bair MJ, Robinson RL, Katon M, Kroenke K. Depression and pain comorbidity. A literature review. Arch Intern Med. 2003; 163(20): 24332445. DOI: 10.1001/archinte.163.20.2433.

75. Keefe FJ, Lumley M, Anderson T, Lynch T, Studts JL, Carson KL. Pain and emotion: new research directions. J Clin Psychol. 2001; 57(4): 587-607. DOI: $10.1002 /$ jclp. 1030.

76. Kerns RD, Sellinger J, Goodin BR. Psychological treatment of chronic pain. Annu Rev Clin Psychol. 2011; 7: 411-434. DOI: 10.1146/annurevclinpsy-090310-120430.

77. Berg-Weger M, Schneider FD. Interdisciplinary collaboration in social work education. J Soc Work Educ. 1998; 34(1): 97-107.

78. Pardavilia MI, Vivar CG. Necesidades de la familia en las unidades de cuidados intensivos. Revisión de la literatura. Enferm Intensiva. 2012; 23(2): 51-67. DOI: 10.1016/j.enfi.2011.06.001.

79. Jones C, Skirrow P, Griffiths RD, Humphris G, Ingleby $\mathrm{S}$, Eddleston $\mathrm{J}$, et al. Post-traumatic stress disorder-related symptoms in relatives of patients following intensive care. Intensive Care Med. 2004; 30(3): 456-460. DOI: 10.1007/s00134-003-2149-5.

80. Holden J, Harrison L, Johnson M. Families, nurses and intensive care patients: a review of the literature. J Clin Nurs. 2002; 11(2): 140-148. DOI: 10.1046/j.1365-2702.2002.00581.x.

81. Rose L, Haslam L, Dale C, Knechtel L, Fraser M, Pinto R, et al. Survey of assessment and management of pain for critically ill adults. Intensive Crit Care Nurs. 2011; 27(3): 121-128. DOI: 10.1016/j. iccn.2011.02.001.

82. Wang HL, Tsai YF. Nurses' knowledge and barriers regarding pain management in intensive care units. J Clin Nurs. 2010; 19(21-22): 3188-3196. DOI: 10.1111/j.1365-2702.2010.03226.x.

83. Gustafsson M, Borglin G. Can a theorybased educational intervention change nurses' knowledge and attitudes concerning cancer pain management? A quasi-experimental design. BMC Health Serv Res. 2013; 13: 328. DOI: 10.1186/1472-6963-13-328.

84. Patiraki EI, Papathanassoglou ED, Tafas C, Akarepi V, Katsaragakis SG, Kampitsi A, et al. A randomized controlled trial of an educational intervention on hellenic nursing staff's knowledge and attitudes on cancer pain management. Eur J Oncol Nurs. 2006; 10(5): 337-352. DOI: http://dx.doi.org/10.1016/j. ejon.2005.07.006.

85. Park JM, Kim JH. Assessment and treatment of pain in adult intensive care unit patients. The Korean $\mathrm{J}$ Crit Care Med. 2014; 29(3): 147-159. DOI: https:// doi.org/10.4266/kjccm.2014.29.3.147. 